\title{
DIFFICULTIES IN FORMULATING AN IMAGINARY-TIME FORMALISM OF TEMPORAL AXIAL GAUGE AT FINITE T
}

\author{
S.M.H. Wong \\ 円LPTHE, Université Paris-Sud, Bâtiment 211, F-91405 Orsay, France
}

\begin{abstract}
Following the successful construction of the finite T real-time formalism in temporal axial gauge, we attempt to further study the equivalent new imaginary-time formalism of James and Landshoff based on the same Hamiltonian approach in the hope that it will provide the answer to Debye screening in QCD. It turns out that, unlike in the real-time case, energy conservation does not hold because of the unusual representation of the longitudinal field forced upon by the Hamiltonian formulation.
\end{abstract}

LPTHE-Orsay 94/114

November 1994 (revised)

${ }^{1}$ Laboratoire associé au Centre National de la Recherche Scientifique 


\section{Introduction}

In our previous attempt [1] to study the screening behaviour of the singlet quarkantiquark potential in a quark-gluon plasma within the framework of Braaten and Pisarski resummed perturbation theory [2] in temporal axial gauge (TAG), we encountered some difficulties in connection with the longitudinal propagator. They arise due to the singular $1 / p_{0}^{2}$ factor at $p_{0}=0$ of the Matsubara frequency sum. These difficulties were

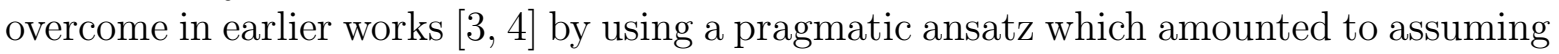
the standard formula [5] for performing the frequency sum

$$
T \sum_{n} f\left(p_{0}\right)=\frac{1}{2 i \pi}\left\{\int_{-i \infty}^{i \infty} \mathrm{d} z f(z)+\int_{-i \infty+\epsilon}^{i \infty+\epsilon} \mathrm{d} z(f(z)+f(-z)) \frac{1}{e^{z / T}-1}\right\}
$$

holds even in the case that $f(z)$ has poles on the imaginary axis. So we have the following vanishing sums

$$
T \sum 1 / p_{0}=0 \quad, \quad T \sum 1 / p_{0}^{2}=0 .
$$

As noted in these papers, there exists no proof that this is correct to any order although at leading order this is found to be correct.

Indeed by using this ansatz, we found, at next to leading order in TAG, a power screening behaviour which is in contradiction with that obtained in covariant gauge and Coulomb gauge [6, 7, 8]. Moreover, it is also in disagreement with the result obtained from the correlator of two Polyakov loops [10, 11]. However, with the use of this ansatz, similar result was also found in TAG by some other unique construction 9].

Instead of using such an ansatz, one can use a general axial gauge $n \cdot A=0$ with a time-like 4-vector $n^{\mu}=(1, \mathbf{n})$ and obtain TAG in the limit $\mathbf{n} \rightarrow 0$. However, the usual simplification of the vanishing space-time, time-time components of the propagator in TAG is gone [12].

It has been pointed out by James and Landshoff [13] that the imaginary-time longitudinal propagator used in the previous works cannot be correct because including only physical states in the thermal average breaks the periodicity of the longitudinal propagator. They have initiated a new formalism in which the longitudinal propagator comes out automatically free of the $p_{0}=0$ singularity. They have shown that one can obtain the same answer of the two-loop pressure in this formalism as in covariant gauge and Feynman gauge [13, 19].

In the hope that this formalism will provide the answer to the above contradiction and difficulties and to see whether the power screening is a physical result or a gauge artifact, we study this new formalism. One striking feature of this formalism is that the simple basic energy conservation mechanism in the usual theory is absent so one may wonder whether energy conservation is still there. We will explain this mechanism in the usual theory and why it is absent in the new formalism in the next section. In section 3, we find the physical states for performing the thermal average and we will show 
explicitly the absence of energy conservation in some one-loop propagators in section 1 . In section 5, we will give an argument for energy conservation and in section 6, we will point out the reason of energy non-conservation.

\section{James and Landshoff's TAG Formalism}

In this section, we describe briefly the formalism of James and Landshoff [13].

At zero temperature, TAG is known to require a prescription to deal with the $1 / p_{0}^{2}$ factor in the longitudinal propagator. It has been the case that Wilson loop calculation becomes the almost standard test for any potentially correct prescription. The Vienna prescription [14] which is a generalization of the Mandelstam-Leibbrandt prescription [15] in light-cone gauge was found to be better than the others such as principal value prescription. It is therefore natural to try to extend this to finite temperature. This has been done by James in real-time [17, 18, 19] and James and Landshoff in imaginary-time [13] based on the Hamiltonian approach to the Vienna prescription derived in [16]. Here we concern ourselves only with the imaginary-time formalism.

With the absence of ghost in TAG, the thermal average has to be taken only over physical states. One immediate consequence is that the longitudinal propagator is not periodic in time despite the fact that gluons are bosons (compared with the usual formalism). This has to do with the fact that whether the propagator of a given particle is periodic depends not only on its species but also on whether it is heated in the heat bath. This peculiar situation happens only in the case of gauge particles due to the Hilbert space occupied by them is not everywhere physical. It is easy to see this in a simplified situation of a free theory. Recall that in quantum mechanics, the amplitude for evolving from a state $Q$ at $T$ to a state $Q^{\prime}$ at $T^{\prime}$ is given by

$$
\left\langle Q^{\prime} T^{\prime} \mid Q T\right\rangle=\int[d Q] \exp \left\{-i \int_{T}^{T^{\prime}} d t L\right\},
$$

so one can convert the partition function for, say scalar theory, into path integral form, i.e.

$$
\begin{aligned}
Z_{\phi} & =\operatorname{tr}\left\langle\phi\left|\exp \left(-\beta H_{\phi}\right)\right| \phi\right\rangle \\
& =\int_{\text {periodic }}[d \phi] \exp \left\{-i \int_{0}^{-i \beta} d t L_{\phi}\right\} .
\end{aligned}
$$

The interpretation is that one starts from a state $\phi$ at $t=0$ and evolves back through a time $-i \beta$ to $\phi$ so that $\phi$ has to be periodic in $-i \beta$. The equivalent for gauge fields in the free field case is

$$
\begin{aligned}
Z_{A} & =\operatorname{tr}\left\langle T\left|\exp \left(-\beta H_{A}\right)\right| T\right\rangle \\
& =\int_{\text {periodic }}\left[d A_{T}\right] \exp \left\{-i \int_{0}^{-i \beta} d t L_{A_{T}}\right\} .
\end{aligned}
$$


Since only physical transverse states are included in the thermal average, the longitudinal field part of the Hamiltonian has only the vacuum to act on so there is no path integral for the longitudinal field. The result is only transverse states need to be periodic in time. There is nothing to say that the longitudinal field has to be so, in contradiction with the usual formalism where all bosonic fields are automatically taken to be periodic.

From the definition of the contour ordered propagator [20], periodic (anti-periodic) fields imply Kubo, Martin, Schwinger condition (KMS) or periodic (anti-periodic) boundary condition for the propagators therefore the transverse gauge propagator and scalar propagator are periodic with period $-i \beta$ but the propagator of the non-periodic longitudinal field has no such periodicity. (Note that the trace identity $\operatorname{tr}(A B)=\operatorname{tr}(B A)$, which we do not have here, is not necessary for the transverse gauge propagator and the scalar propagator to be periodic.)

Because of the absence of periodicity in $-i \beta$, the longitudinal propagator, of which the time-momentum representation is given by [13]

$$
D_{a b}^{L}(t, \mathbf{k})_{i j}=i \delta_{a b} \frac{k_{i} k_{j}}{\mathbf{k}^{2}} \theta\left(-k_{3} \operatorname{Im} t\right)|\operatorname{Im} t|
$$

when Fourier transformed using the full time range, i.e. between $-i \beta$ and $i \beta$, into imaginary energy space has bosonic as well as fermionic mode. It is now not obvious how energy is conserved in this formalism. Remember in the usual case, at each interaction vertex, take three particles interaction for example, one has the following Euclidean time integral

$$
\int_{0}^{\beta} \mathrm{d} \tau \exp \left\{i\left(p_{1}^{0}+p_{2}^{0}+p_{3}^{0}\right) \tau\right\}
$$

where $p_{i}^{0}, i=1,2,3$ are the integral multiple $\pi T$ valued energies entering the vertex. For the case of three "usual" bosons, this integral gives a kronecker-delta, because the sum of the energies is even, which ensures energy conservation. For the case of a "usual" bosonfermions interaction, because of charge conservation, there must be a pair of fermions interacting with one "usual" boson, so the energy sum is still even and again we have energy conservation. But in the case of this new formalism, longitudinal gluon or photon can have either even or odd integral multiple of $\pi T$ as energy, so now the energy sum at the vertex needs not be even therefore we may or may not get a kronecker-delta to ensure energy conservation. The obvious question to ask is how is energy conserved. We will show that this is indeed a problem of this formalism when we study some one-loop propagators in the abelian theory in the following sections. Before one can do that, one will have to find out the physical states to do thermal average over. This is due to the fact that Gauss' law is lost when one chooses to work in TAG. One finds the physical states by requiring

$$
\left\langle p h y s\left|\exp \left(\int \mathrm{d}^{3} \mathbf{x} \omega^{a} \lambda^{a}\right)\right| p h y s^{\prime}\right\rangle=\left\langle p h y s \mid p h y s^{\prime}\right\rangle,
$$


where $\lambda^{a}$ 's are the Gauss' laws and $\omega^{a}$ 's are just devices introduced to sum over colours. Their actual values are unimportant since what matters is the matrix elements of any power of Gauss' law between physical states vanish. This will be dealt with in detail in section 3 .

\section{In search of $\mathcal{O}$}

In James and Landshoff's original paper, it was shown that because of the Euclidean time range being finite (from 0 to $\beta$ ), one cannot form matrix elements between free incoming and/or outgoing states. One is not allowed to switch off interactions at finite time without changing the theory completely. So one has to find the physical states at a certain time, say $t=0$, in order to find thermal averages. They advocate an expansion of the form

$$
|p h y s\rangle=\mathcal{O}|T\rangle
$$

where $|T\rangle$ are the free transverse gluon states and $\mathcal{O}$ is an operator series

$$
\mathcal{O}=1+e o_{1}+e^{2} o_{2}+e^{3} o_{3}+\cdots
$$

so $|T\rangle$ are the physical states when $e=0$.

The operators $o_{i}$ are obtained by requiring the matrix elements of any power of Gauss's law between physical states to be zero to make up for the loss of Gauss' law in TAG. We shall see that it is a very weak condition to find physical states. Gauss' law in a non-abelian pure gauge theory in TAG is

$$
\lambda^{a}(t, \mathbf{x})=\boldsymbol{\partial} \cdot \dot{\mathbf{A}}^{a}(t, \mathbf{x})-g f^{a b c} \mathbf{A}^{b}(t, \mathbf{x}) \cdot \dot{\mathbf{A}}^{c}(t, \mathbf{x}) .
$$

James and Landshoff found that it was not sufficient to apply Gauss's law once but must do so repeatedly to find the $o_{1}$. To simplify matters, we will study $\mathcal{O}$ in an abelian theory. It turns out this can be solved completely in this case.

\section{$3.1 \mathcal{O}$ in Abelian Theories}

In this section, we will study scalar electrodynamics but the same also holds for QED as far as finding $\mathcal{O}$ is concerned. In scalar electrodynamics, the simplest langrangian is given by (including $\phi^{3}$ or $\phi^{4}$ type self-interaction will obviously not affect $\mathcal{O}$ )

$$
\mathcal{L}=\int \mathrm{d}^{3} \mathbf{x}\left\{\left(\partial_{\mu}-i e A_{\mu}\right) \phi\right\}^{\dagger}\left\{\left(\partial_{\mu}-i e A_{\mu}\right) \phi\right\}-\frac{1}{4} \int \mathrm{d}^{3} \mathbf{x} F^{\mu \nu} F_{\mu \nu}
$$

so Gauss' law in TAG is

$$
\partial \cdot \dot{\mathbf{A}}-i e\left(\dot{\phi}^{\dagger} \phi-\phi^{\dagger} \dot{\phi}\right)=0
$$


From now on we will write

$$
\Phi=\left(\dot{\phi}^{\dagger} \phi-\phi^{\dagger} \dot{\phi}\right),
$$

and before we solve for $\mathcal{O}$, we will split each $o_{i}$ into two parts

$$
o_{i}=o_{i}^{A}+o_{i}^{H}
$$

for the anti-Hermitian part and Hermitian part of $o_{i}$ respectively.

At order $e^{1}$, we have to solve

$$
\left\langle T \phi\left|\left[\boldsymbol{\partial} \cdot \dot{\mathbf{A}}, o_{1}^{A}\right]+\left\{\boldsymbol{\partial} \cdot \dot{\mathbf{A}}, o_{1}^{H}\right\}-i \Phi\right| T^{\prime} \phi^{\prime}\right\rangle=0 .
$$

Here the states are free transverse photon states and free scalar states. If we assume

$$
\left\langle T \phi\left|\left[\boldsymbol{\partial} \cdot \dot{\mathbf{A}}, o_{1}^{A}\right]-i \Phi\right| T^{\prime} \phi^{\prime}\right\rangle=0,
$$

then we have, using the commutation relations in [13], 16], the solution

$$
{ }^{\lambda} O_{1}^{A}=\int \mathrm{d}^{3} \mathbf{x} \frac{1}{\boldsymbol{\partial}^{2}} \boldsymbol{\partial} \cdot \mathbf{A}(x) \Phi(x) .
$$

The superscript $\lambda$ is to remind ourselves that this is the solution of $o_{1}^{A}$ to using Gauss' law once. The remaining equation

$$
\left\langle T \phi\left|\left\{\boldsymbol{\partial} \cdot \dot{\mathbf{A}}, o_{1}^{H}\right\}\right| T^{\prime} \phi^{\prime}\right\rangle=0,
$$

does not define $o_{1}^{H}$ very well. $o_{1}^{H}$ can be a term with no $\boldsymbol{\partial} \cdot \mathbf{A}$ or product of more than one $\boldsymbol{\partial} \cdot \mathbf{A}$ or term with at least one $\boldsymbol{\partial} \cdot \dot{\mathbf{A}}$. They all satisfy the above equation. Applying Gauss' law repeatedly does not uniquely determine $o_{1}^{H}$ although it does eliminate some possibilities. The same can also be said about $o_{1}^{A}$. One can easily add a term to Eq. (18) and still satisfies Eq. (17). We will aim at finding the simplest solution of $\mathcal{O}$ to the Gauss' law constraint so we do not put an unknown term to Eq. (18) in the hope that we might be able to solve for it later. Also the simplest solution for $o_{1}^{H}$ is zero. We will do this as long as we do not run into equations that cannot be solved. In that case, it could be a sign that extra terms are needed. It turns out that in an abelian theory such as scalar electrodynamics or QED, it works. But in non-abelian theories, it is much more difficult, and we will not go into this case in this work. In our present case, we set

$$
{ }^{\lambda} O_{1}^{H}=0 .
$$

Applying Gauss' law twice at order $e^{1}$, we have

$$
\begin{aligned}
\langle T \phi|\left[\{\boldsymbol{\partial} \cdot \dot{\mathbf{A}}(x), \boldsymbol{\partial} \cdot \dot{\mathbf{A}}(y)\}, o_{1}^{A}\right]+\left\{\{\boldsymbol{\partial} \cdot \dot{\mathbf{A}}(x), \boldsymbol{\partial} \cdot \dot{\mathbf{A}}(y)\}, o_{1}^{H}\right\} \\
-i\{\Phi(x), \boldsymbol{\partial} \cdot \dot{\mathbf{A}}(y)\}-i\{\Phi(y), \boldsymbol{\partial} \cdot \dot{\mathbf{A}}(x)\}\left|T^{\prime} \phi^{\prime}\right\rangle=0 .
\end{aligned}
$$


One easily sees that using

$$
\left[o_{1}^{A},\{\boldsymbol{\partial} \cdot \dot{\mathbf{A}}(x), \boldsymbol{\partial} \cdot \dot{\mathbf{A}}(y)\}\right]=\left\{\left[o_{1}^{A}, \boldsymbol{\partial} \cdot \dot{\mathbf{A}}(x)\right], \boldsymbol{\partial} \cdot \dot{\mathbf{A}}(y)\right\}+\left\{\boldsymbol{\partial} \cdot \dot{\mathbf{A}}(x),\left[o_{1}^{A}, \boldsymbol{\partial} \cdot \dot{\mathbf{A}}(y)\right]\right\},
$$

the simplest solution at one $\lambda$ level Eq. (18) and Eq. (20) also holds here. That is ${ }^{\lambda} O_{1}^{A}={ }^{\lambda^{2}} O_{1}^{A}$ and ${ }^{\lambda} O_{1}^{H}={ }^{\lambda^{2}} O_{1}^{H}$.

Perhaps one should note that

$$
[\Phi(x), \Phi(y)]=0
$$

and is essentially the reason why an abelian theory is so much simpler. The equivalence in QED is to replace $\Phi$ in Eq. (18) by $\bar{\psi} \gamma^{0} \psi$ and we obviously have

$$
\left[\bar{\psi}(x) \gamma^{0} \psi(x), \bar{\psi}(y) \gamma^{0} \psi(y)\right]=0 \text {. }
$$

It is now clear that the solution Eq. (18) and Eq. (20) will satisfy the Gauss' law constraint at order $e^{1}$ no matter how many times it is applied. Henceforth the $\lambda$ superscript of $o_{1}$ will be dropped.

At order $e^{2}$, applying Gauss' law once gives

$$
\left\langle T \phi\left|\left[\boldsymbol{\partial} \cdot \dot{\mathbf{A}}, o_{2}^{A}\right]+\left\{\boldsymbol{\partial} \cdot \dot{\mathbf{A}}, o_{2}^{H}\right\}-i\left[\Phi, o_{1}^{A}\right]+o_{1}^{\dagger} \boldsymbol{\partial} \cdot \dot{\mathbf{A}} o_{1}\right| T^{\prime} \phi^{\prime}\right\rangle=0 .
$$

The last two terms are vanishing and the remaining two terms cannot cancel each other and have to vanish individually. So

$$
\lambda_{O_{2}}=0
$$

is the simplest.

At the $\lambda^{2}$ level, after some rearrangement, we get

$$
\left\langle T \phi\left|\left[\{\boldsymbol{\partial} \cdot \dot{\mathbf{A}}(x), \boldsymbol{\partial} \cdot \dot{\mathbf{A}}(y)\}, o_{2}^{A}\right]+\left\{o_{2}^{H}-\frac{1}{2}\left(o_{1}^{A}\right)^{2},\{\boldsymbol{\partial} \cdot \dot{\mathbf{A}}(x), \boldsymbol{\partial} \cdot \dot{\mathbf{A}}(y)\}\right\}\right| T^{\prime} \phi^{\prime}\right\rangle=0 .
$$

The simplest solutions at this level are then

$$
o_{2}^{A}=0, \quad o_{2}^{H}=\frac{1}{2}\left(o_{1}^{A}\right)^{2} .
$$

These, in fact, satisfy all order $e^{2}$ Gauss' law constraints that is why no superscript $\lambda^{2}$ has been given to them. One can go on and it becomes clear that

$$
o_{3}^{A}=\frac{1}{3 !}\left(o_{1}^{A}\right)^{3}, \quad o_{3}^{H}=0 .
$$

So we find, for scalar electrodynamics or QED,

$$
\begin{aligned}
\mathcal{O} & =1+e o_{1}^{A}+\frac{1}{2 !}\left(e o_{1}^{A}\right)^{2}+\frac{1}{3 !}\left(e o_{1}^{A}\right)^{3}+\ldots \\
& =\exp \left(e o_{1}^{A}\right) .
\end{aligned}
$$


In scalar electrodynamics $o_{1}^{A}$ is given by Eq. (18) whereas in QED, it is

$$
o_{1 Q E D}^{A}=i \int \mathrm{d}^{3} \mathbf{x} \frac{1}{\boldsymbol{\partial}^{2}} \boldsymbol{\partial} \cdot \mathbf{A}(x) \bar{\psi}(x) \gamma^{0} \psi(x)
$$

So we find that $\mathcal{O}$ is unitary and therefore if the $\left|T^{\prime} \phi^{\prime}\right\rangle$ is normalized so is $\mathcal{O}\left|T^{\prime} \phi^{\prime}\right\rangle$. With these results, we can now investigate the one-loop propagator.

\section{One-loop Propagator in the Abelian Theory}

In this section, we investigate the problem of energy conservation in TAG in this formalism which has already been allured to in the previous sections. We do that by looking at the one-loop propagators in scalar electrodynamics. Obviously, the propagator for the transverse gluon is energy conserving at the one-loop level since both the scalar field and the transverse propagator remain the same as in the usual formalism. The same is also true for the one-loop scalar propagator formed with a transverse gluon internal line. So we start by studying the scalar propagator with a longitudinal gluon internal line.

In this formalism, there are four diagrams that contribute to the one-loop scalar propagator with a longitudinal gluon line, $\mathcal{D}_{l}^{\phi}$. The diagram with no $o_{1}$ vertex, with one $o_{1}^{\dagger}(-i \beta)$ at the left vertex, with one $o_{1}(0)$ at the right vertex and the one with two $o_{1}$ 's i.e. with $o_{1}^{\dagger}(-i \beta)$ at the left and $o_{1}(0)$ at the right vertex as shown in Fig. 1. (The diagrams constructed from the $e^{2}$ term of $\mathcal{O}$, that is those with two $o_{1}(0)$ 's or with two $o_{1}^{\dagger}(-i \beta)$ 's are zero because $D^{L}(t=0)=0$.) We will let the Euclidean times $\tau_{1}$ and $\tau_{2}$ to be the time integration variables at the left and at the right vertex respectively.

Combining the four diagrams, we have the following expression

$$
\begin{aligned}
& \mathcal{D}_{l}^{\phi}\left(s_{1}, s_{2}, \mathbf{k}\right)=e^{2} \int \frac{\mathrm{d}^{3} \mathbf{q}}{(2 \pi)^{3}} \quad \int \frac{\mathrm{d}^{3} \mathbf{p}}{(2 \pi)^{3}}(2 \pi)^{3} \delta(\mathbf{p}+\mathbf{q}+\mathbf{k}) \frac{1}{\mathbf{q}^{2}} T^{3} \sum_{k_{10}, k_{20}, p_{0}} \frac{1}{K_{1}^{2} K_{2}^{2} P^{2}} \\
& \int_{0}^{\beta} \mathrm{d} \tau_{1} \int_{0}^{\tau_{1}} \mathrm{~d} \tau_{2}\left\{\left(\mathbf{k}^{2}-\mathbf{p}^{2}\right)^{2}+i\left(p_{0}+k_{10}\right)\left(\mathbf{k}^{2}-\mathbf{p}^{2}\right) \delta\left(\beta-\tau_{1}\right)+i\left(p_{0}+k_{20}\right)\left(\mathbf{k}^{2}-\mathbf{p}^{2}\right) \delta\left(\tau_{2}\right)\right. \\
& \left.+\left(\mathbf{p}^{2}+i p_{0} i\left(k_{10}+k_{20}\right)+i k_{10} i k_{20}\right) \delta\left(\beta-\tau_{1}\right) \delta\left(\tau_{2}\right)\right\} \\
& e^{i p_{0}\left(\tau_{1}-\tau_{2}\right)} e^{-i k_{10}\left(\tau_{1}-\sigma_{1}\right)} e^{-i k_{20}\left(\sigma_{2}-\tau_{2}\right)} D^{L}\left(t_{1}-t_{2}, \mathbf{q}\right)
\end{aligned}
$$

where $k_{10}$ and $k_{20}$ are the incoming (from the left) and outgoing energies (to the right) respectively, $K_{i}^{2}=\left(i k_{i 0}\right)^{2}-\mathbf{k}^{2}, P^{2}=\left(i p_{0}\right)^{2}-\mathbf{p}^{2}$, and $t_{i}=-i \tau_{i}, i=1,2$ and $\sigma_{i}$ 's are the Euclidean external times (that is this propagator starts at $s_{1}=-i \sigma_{1}$ and ends at $\left.s_{2}=-i \sigma_{2}\right)$. We are restricting ourselves to the integration range in which $D^{L}$ will give $\theta\left(q_{3}\right)$. The other integration range will give the same answer but with $\theta\left(-q_{3}\right)$ so the sum is independent of the direction of $q_{3}$. 
We first convert the energy sum over $p_{0}$ into contour integral and then use the relation

$$
\delta(\tau)=\frac{\mathrm{d}}{\mathrm{d} \tau} \theta(\tau)
$$

and partial integration over $\tau$ to remove the delta functions. The surface terms are vanishing either because

$$
D^{L}(0, \mathbf{q})=0 .
$$

or because

$$
\theta(0)=0,
$$

which we choose to save on algebra and it has to hold for consistency (see appendix A.1) if one uses

$$
\int_{0}^{\beta} \mathrm{d} \tau \delta(\tau)=1 .
$$

One can of course choose the more conventional $\theta(0)=\frac{1}{2}$ but then one will have to use

$$
\int_{0}^{\beta} \mathrm{d} \tau \delta(\tau)=\frac{1}{2} .
$$

With this latter choice, the surface terms do not go away but the final result is the same. After some algebra, using

$$
T \sum_{p_{0}} \exp \left\{i p_{0}\left(\tau-\tau^{\prime}\right)\right\}=\delta\left(\tau-\tau^{\prime}\right)
$$

and

$$
\frac{\mathrm{d}}{\mathrm{d} \tau_{1}} \frac{\mathrm{d}}{\mathrm{d} \tau_{2}} D_{L}\left(t_{1}-t_{2}, \mathbf{q}\right)=-i \delta\left(\tau_{1}-\tau_{2}\right),
$$

we get

$$
\begin{aligned}
\mathcal{D}_{l}^{\phi}\left(s_{1}, s_{2}, \mathbf{k}\right)= & e^{2} \int \frac{\mathrm{d}^{3} \mathbf{q}}{(2 \pi)^{3}} \int \frac{\mathrm{d}^{3} \mathbf{p}}{(2 \pi)^{3}}(2 \pi)^{3} \delta(\mathbf{p}+\mathbf{q}+\mathbf{k}) \frac{1}{\mathbf{q}^{2}} \sum_{s= \pm 1} \frac{s N(s|\mathbf{p}|)}{2|\mathbf{p}|} i \theta\left(q_{3}\right) \\
& \left\{-T \sum_{k_{20}} \frac{\left(i k_{20}+s|\mathbf{p}|\right)}{K_{2}^{2}}\left(\frac{e^{i k_{20}\left(\sigma_{1}-\sigma_{2}\right)}-e^{-i k_{20} \sigma_{2}+s|\mathbf{p}| \sigma_{1}}}{i k_{20}-s|\mathbf{p}|}\right)\right. \\
& -T \sum_{k_{10}} \frac{\left(i k_{10}+s|\mathbf{p}|\right)}{K_{1}^{2}}\left(\frac{e^{i k_{10}\left(\sigma_{1}-\sigma_{2}\right)}-e^{i k_{10} \sigma_{1}+s|\mathbf{p}|\left(\beta-\sigma_{2}\right)}}{i k_{10}-s|\mathbf{p}|}\right) \\
& \left.+T^{2} \sum_{k_{10}, k_{20}} \frac{\left(s|\mathbf{p}|+i k_{10}\right)\left(s|\mathbf{p}|+i k_{20}\right)}{K_{1}^{2} K_{2}^{2}} \beta \delta_{k_{10}, k_{20}} e^{i k_{10}\left(\sigma_{1}-\sigma_{2}\right)}\right\},
\end{aligned}
$$

where $N(|\mathbf{p}|)$ is the Bose-Einstein distribution function. We see that in the first two terms, the last term within each round brackets do not have time-translation invariance. This happens when the poles at $\pm|\mathbf{k}|$ are picked up by the contour integrals which replace 
the energy sums. We now show that neither the one-loop longitudinal propagator, $\mathcal{D}^{L}$, has time-translation invariance.

We again have four diagrams to consider plus the tadpole graph. (Again diagrams constructed with two $o_{1}(0)$ 's or two $o_{1}^{\dagger}(-i \beta)$ 's are zero. This time, this is due to the product of the opposing theta functions $\theta\left(k_{3}\right) \theta\left(-k_{3}\right)$ coming from the longitudinal propagators of the external lines.) Ignoring the tadpole for the time being and with the same arrangement of the $o_{1}$ vertices as in the previous case, Fig. 2, we get

$$
\begin{aligned}
& \mathcal{D}_{i j}^{L}\left(s_{1}, s_{2}, \mathbf{k}\right)=-e^{2} \int \frac{\mathrm{d}^{3} \mathbf{q}}{(2 \pi)^{3}} \int \frac{\mathrm{d}^{3} \mathbf{p}}{(2 \pi)^{3}} \quad(2 \pi)^{3} \delta(\mathbf{p}+\mathbf{q}+\mathbf{k}) \frac{k_{i} k_{j}}{\left(\mathbf{k}^{2}\right)^{2}} T^{2} \sum_{p_{0}, q_{0}} \frac{1}{P^{2} Q^{2}} \\
& \int_{\sigma_{1}}^{\beta} d \tau_{1} \int_{0}^{\sigma_{2}} d \tau_{2}\left\{\left(\mathbf{p}^{2}-\mathbf{q}^{2}\right)^{2}+i\left(p_{0}-q_{0}\right)\left(\mathbf{p}^{2}-\mathbf{q}^{2}\right)\left(\delta\left(\beta-\tau_{1}\right)+\delta\left(\tau_{2}\right)\right)\right. \\
& \left.+\left(\mathbf{p}^{2}+\mathbf{q}^{2}-2 i p_{0} i q_{0}\right) \delta\left(\beta-\tau_{1}\right) \delta\left(\tau_{2}\right)\right\} \quad e^{-i\left(p_{0}+q_{0}\right)\left(\tau_{1}-\tau_{2}\right)} D^{L}\left(t_{1}-s_{1}, \mathbf{k}\right) D^{L}\left(s_{2}-t_{2}, \mathbf{k}\right),
\end{aligned}
$$

where $s_{i}=-i \sigma_{i}, i=1,2$ and again we take $\sigma_{2}>\sigma_{1}$. We evaluate the energy sums by contour integrals, this requires us to split the time integrals into various range and switching $p_{0}, q_{0} \rightarrow-p_{0},-q_{0}$ if necessary for convergence at infinity on the complex $p_{0}$ and $q_{o}$ energy planes. After some cancellation between the various graphs we get

$$
\begin{aligned}
& \mathcal{D}_{i j}^{L}\left(s_{1}, s_{2}, \mathbf{k}\right)=e^{2} \int \frac{\mathrm{d}^{3} \mathbf{q}}{(2 \pi)^{3}} \int \frac{\mathrm{d}^{3} \mathbf{p}}{(2 \pi)^{3}}(2 \pi)^{3} \delta(\mathbf{p}+\mathbf{q}+\mathbf{k}) \frac{k_{i} k_{j}}{\left(\mathbf{k}^{2}\right)^{2}} \sum_{r, s= \pm 1} \frac{r N(r|\mathbf{p}|)}{2|\mathbf{p}|} \frac{s N(s|\mathbf{q}|)}{2|\mathbf{q}|} \theta\left(k_{3}\right) \\
& \left(\mathbf{p}^{2}-\mathbf{q}^{2}\right)^{2}\left\{\frac{1}{(r|\mathbf{p}|+s|\mathbf{q}|)^{4}}-\frac{2\left(\sigma_{2}-\sigma_{1}\right)}{(r|\mathbf{p}|+s|\mathbf{q}|)^{3}}-\frac{\left(\sigma_{2}-\sigma_{1}\right)^{3}}{3(r|\mathbf{p}|+s|\mathbf{q}|)}+\frac{e^{(r|\mathbf{p}|+s|\mathbf{q}|)\left(\sigma_{2}-\sigma_{1}\right)}}{(r|\mathbf{p}|+s|\mathbf{q}|)^{4}}\right. \\
& \left.-\frac{e^{(r|\mathbf{p}|+s|\mathbf{q}|) \sigma_{2}}}{(r|\mathbf{p}|+s|\mathbf{q}|)^{4}}-\frac{e^{(r|\mathbf{p}|+s|\mathbf{q}|) \sigma_{1}}}{(r|\mathbf{p}|+s|\mathbf{q}|)^{4}}\right\} \text {. }
\end{aligned}
$$

The last two terms explicitly do not have time-translation invariance.

One can of course verify that this result is correct by evaluating the time integrals first before doing the energy sums but more interestingly a simpler check can be performed in the special case of $\sigma_{1}=\sigma_{2}=\sigma$. In Eq. (41), we can now evaluate the energy sums and there is no need to split up the time integrals into various range. Furthermore, one can now use Eq. (33), Eq. (34) and Eq. (35) to have some quick cancellations because the surface terms can again be made to vanish. Doing it this way, one gets

$$
2(r|\mathbf{p}|-s|\mathbf{q}|)^{2} \frac{1-e^{(r|\mathbf{p}|+s|\mathbf{q}|) \sigma}}{(r|\mathbf{p}|+s|\mathbf{q}|)^{2}}
$$

for the whole factor of the last two lines of Eq. (42) which agrees with that in that equation when $\sigma_{1}=\sigma_{2}=\sigma$. We stress again that this result is independent of the exact 
choice of the value of the theta function at zero. We choose it to vanish at that point only for convenience.

One may wonder in this case, the terms without time-translation invariance may be canceled by those coming from the tadpole graph. Unfortunately, this is not the case. The contribution from the tadpole, Fig. 3, preserves time-translation invariance and is therefore energy conserving. It is

$$
\mathcal{D}_{\text {ij tadpole }}^{L}\left(s_{1}, s_{2}, \mathbf{k}\right)=e^{2} T \sum_{p_{0}} \int \frac{\mathrm{d}^{3} \mathbf{p}}{(2 \pi)^{3}} \frac{k_{i} k_{j}}{\mathbf{k}^{2}} \frac{1}{P^{2}} \int_{0}^{\beta} \mathrm{d} \tau D^{L}\left(t-s_{1}, \mathbf{k}\right) D^{L}\left(s_{2}-t, \mathbf{k}\right) .
$$

The time integral give

$$
\frac{\left(\sigma_{2}-\sigma_{1}\right)^{3}}{6}
$$

Therefore the complete one-loop longitudinal propagator has no energy conservation.

\section{An Argument for Energy Conservation}

In this section, we will give an argument for energy conservation and therefore timetranslation invariance of any thermal N-point function in order to better explain the reason for the counter examples of the one-loop propagators that we have found above.

Thermal averages of an operator $Q$ expressed in the interaction picture is

$$
\langle Q(t)\rangle=Z^{-1} \sum_{p h y s}\left\langle p h y s\left|\exp \left(-\beta H_{0 I}\right) U(-i \beta, t) Q_{I}(t) U(t, 0)\right| p h y s\right\rangle,
$$

where the sum is over physical states and we have chosen $t=0$ to be the time when the interaction picture coincides with the Heisenberg picture. Therefore a general N-point function in scalar electrodynamics has the form

$$
\left\langle A\left(t_{1}\right) \phi\left(t_{2}\right) \ldots A\left(t_{n}\right)\right\rangle=Z^{-1} \sum_{\text {phys }}\left\langle p h y s\left|\exp \left(-\beta H_{0 I}\right) B_{n}\left(t_{1}, t_{2}, \ldots, t_{n}\right)\right| p h y s\right\rangle,
$$

where we have assumed $t_{1}>t_{2}>\ldots>t_{n}$ and

$$
B_{n}\left(t_{1}, t_{2}, \ldots, t_{n}\right)=U\left(-i \beta, t_{1}\right) A_{I}\left(t_{1}\right) U\left(t_{1}, t_{2}\right) \phi_{I}\left(t_{2}\right) U\left(t_{2}, t_{3}\right) \cdots A_{I}\left(t_{n}\right) U\left(t_{n}, 0\right) .
$$

The fields here can be any combination of gauge fields and scalar fields provided we have charge conservation.

In particular, the 2-point gauge function is

$$
\begin{aligned}
& \left\langle A\left(t_{1}\right) A\left(t_{2}\right)\right\rangle \\
= & Z^{-1} \sum_{p h y s}\left\langle p h y s\left|\exp \left(-\beta H_{0 I}\right) B_{2}\left(t_{1}, t_{2}\right)\right| p h y s\right\rangle \\
= & Z^{-1} \sum_{\text {phys }}\left\langle p h y s\left|\exp (-i H \delta) \exp \left(-\beta H_{0 I}\right) B_{2}\left(t_{1}+\delta, t_{2}+\delta\right) \exp (i H \delta)\right| p h y s\right\rangle .
\end{aligned}
$$


The second line here is obtained by using the equation

$$
Q_{I}(t+\delta)=\exp \left(i H_{0 I} \delta\right) Q_{I}(t) \exp \left(-i H_{0 I} \delta\right)
$$

for an operator $Q$ in the interaction picture.

Had we been working with a non-gauge theory, we could have inserted two complete sets of states into Eq. (50) and removed the factors $\exp ( \pm i H \delta)$. Or equivalently, using the trace identity $\operatorname{tr}(A B)=\operatorname{tr}(B A)$. But since in a gauge theory, part of the Hilbert space is not physical and we are working in a ghostless gauge, this trick is no longer available to us. Instead we argue that since the physical states $|p h y s\rangle$ can be expressed in terms of energy eigenstates of the full Hamiltonian, the exponentials with the full Hamiltonian $H$ at either end of the sum over physical states of Eq. (50) cancel each other. So we have

$$
\left\langle A\left(t_{1}\right) A\left(t_{2}\right)\right\rangle=\left\langle A\left(t_{1}+\delta\right) A\left(t_{2}+\delta\right)\right\rangle .
$$

The same also applies to the scalar two-point function. Time translation invariance means energy conservation, therefore the 2-point function is energy conserved in momentum space. In other words, the energy coming in equals to the one going out of the propagator. This obviously also works for any N-point function so we should have total sum of energies going into a graph to be zero. This conclusion obviously contradicts what we have found in section 4 , so what is wrong? To understand exactly what is going on, one will have to study the longitudinal gauge field and the Hamiltonian which are so constructed to yield the Vienna prescription in the zero temperature longitudinal propagator. This leads us to the next section.

\section{The Longitudinal Gauge Field and The Hamilto- nian of James and Landshoff's Formalism}

We will point out the unusual features and peculiarities of James and Landshoff's longitudinal field and Hamiltonian in this section and give an explanation of the contradiction that we have found previously.

In order to arrive at the Vienna prescription, the choice of [16]

$$
A_{0}=\frac{\eta}{\partial_{3}} \frac{1}{\partial^{2}} \partial \cdot \mathbf{A}
$$

was made. The Hamiltonian formulation then forces the longitudinal field to have four annihilation and creation operators $q(\mathbf{k}), q^{\dagger}(\mathbf{k}), p(\mathbf{k}), p^{\dagger}(\mathbf{k})$ as opposed to the usual two per field ?. They satisfy

$$
\left[q(\mathbf{k}), p^{\dagger}\left(\mathbf{k}^{\prime}\right)\right]=\mathbf{k}^{2}(2 \pi)^{3} \delta\left(\mathbf{k}-\mathbf{k}^{\prime}\right)
$$

\footnotetext{
${ }^{2}$ For the explicit expression of the longitudinal field written in terms of these operators, we refer to [13, 16.
} 
and its Hermitian conjugate. All other commutation relations between them are vanishing. Observe that in the usual case of two operators per field, one has

$$
\left[a(\mathbf{k}), a^{\dagger}\left(\mathbf{k}^{\prime}\right)\right]=2 k_{0}(2 \pi)^{3} \delta\left(\mathbf{k}-\mathbf{k}^{\prime}\right) .
$$

The change from $2 k_{0}$ to $\mathbf{k}^{2}$ is unimportant here, it is just a matter of dimensions. What is important is the operators commute with their Hermitian conjugates which is not the usual case. Therefore one can construct states using the $q^{\dagger}$ and the $p^{\dagger}$ which have zero norm. For example, the simplest zero norm states are

$$
q^{\dagger}(\mathbf{k})|0\rangle \quad \text { and } \quad p^{\dagger}(\mathbf{k})|0\rangle \text {. }
$$

So the completeness of states is constructed by Dirac's bra and ket state vectors not only of the usual form where the bra is related to the ket by a Hermitian conjugate, eg. $|\mathbf{k}\rangle\langle\mathbf{k}|$, but also of the form where they are not related by such a relation, eg. $q^{\dagger}\left(\mathbf{k}^{\prime}\right)|\mathbf{k}\rangle\langle\mathbf{k}| p\left(\mathbf{k}^{\prime}\right)[16]$.

The longitudinal part of the normal ordered free Hamiltonian in terms of these operators is 16

$$
H_{0}^{L}=\int \frac{\mathrm{d}^{3} \mathbf{k}}{(2 \pi)^{3}} \frac{\theta\left(k_{3}\right)}{\mathbf{k}^{2}}\left\{p^{\dagger}(\mathbf{k}) p(\mathbf{k})+\frac{\eta}{k_{3}}\left(p^{\dagger}(\mathbf{k}) q(\mathbf{k})+q^{\dagger}(\mathbf{k}) p(\mathbf{k})\right)\right\},
$$

so that we have by acting this on the simplest states above

$$
\begin{aligned}
& H_{0}^{L} q^{\dagger}(\mathbf{k})|0\rangle=\left(p^{\dagger}(\mathbf{k})+\frac{\eta}{k_{3}} q^{\dagger}(\mathbf{k})\right)|0\rangle \\
& H_{0}^{L} p^{\dagger}(\mathbf{k})|0\rangle=\frac{\eta}{k_{3}} p^{\dagger}(\mathbf{k})|0\rangle .
\end{aligned}
$$

So the state created by $q^{\dagger}$ is not an energy eigenstate of $H_{0}^{L}$. One cannot construct an energy eigenstate by some combination of it with the state created by $p^{\dagger}$. This is not a problem in the free theory because such states are not physical either because of the Gauss' law constraint or because of the zero norm. They live outside the physical part of the Hilbert space. The problem comes when one turns on the interaction. The physical Hilbert space is now extended somewhat into the previously non-physical part because the interaction Hamiltonian forms a "bridge" linking the two parts.

One can ask whether the above features have any effect on the interacting theory in particular the energy eigenstates. The answer is yes and we will now show it explicitly by constructing energy eigenstates.

Since the part of the interacting Hamiltonian which links the physical Hilbert space in the non-interacting theory to itself produces nothing unusual, we will leave it out. We concentrate only on that part which bridges the physical and the non-physical part and only to first order in $e$. That is we consider $H=H_{0}+e H_{I}$ where $H_{I}$ is

$$
H_{I}=-i \int \mathrm{d}^{3} \mathbf{x} \frac{1}{\partial^{2}} \boldsymbol{\partial} \cdot \mathbf{A}\left(\frac{\mathrm{d}}{\mathrm{d} t}-\frac{\eta}{\partial_{3}}\right)\left(\phi^{\dagger} \dot{\phi}-\dot{\phi}^{\dagger} \phi\right) \text {. }
$$


We treat $H_{I}$ as a small perturbation to the free Hamiltonian $H_{0}$. The states at zero order in $e$ are the free scalar and transverse photon states, i.e.

$$
H_{0}|\phi T\rangle=E_{\phi T}|\phi T\rangle
$$

From order $e^{1}$ and above, the corrections to the free states are constructed from the whole Hilbert space, i.e. the order $e^{n}$ correction to the perturbed free state $|\phi T\rangle$ is of the form

$$
|\phi T\rangle_{p e r t}^{(n)}=\sum_{\alpha} C_{\alpha}^{(n)}|\alpha\rangle
$$

where the sum over $\alpha$ includes the integrals over momenta, sum over spin and other quantum numbers etc. and goes over all states in the Hilbert space. $H_{I}$ will automatically pick out those states which become physical and leave out those that remain non-physical. We choose to work out the energy eigenstates at $\mathrm{t}=0$ when $A^{L}$ contains only $q$ and $q^{\dagger}$ and not $p$ and $p^{\dagger}$.

To understand the following, it is only necessary to know which matrix elements of $H_{I}$ is zero and which is not. Since $H_{I}$ is linear in $A_{L}$, at $\mathrm{t}=0$, the only non-vanishing matrix elements are those between a normal bra state and a suitable 3 ket state which contains one $p^{\dagger}(\mathbf{k})$, and also of course their complex conjugate.

From standard quantum mechanics perturbation theory, we know that the first order perturbed energy is

$$
E_{\phi T}^{(1)}=\left\langle\phi T\left|H_{I}\right| \phi T\right\rangle
$$

(which is zero in this case) and the perturbed state to order $e^{1}$ is

$$
|\phi T\rangle_{\text {pert }}=|\phi T\rangle+e \sum_{\phi^{\prime}} \int \frac{\mathrm{d}^{3} \mathbf{k}}{(2 \pi)^{3}} \frac{\theta\left(k_{3}\right)}{\mathbf{k}^{2}} \frac{q^{\dagger}(\mathbf{k})\left|\phi^{\prime} T\right\rangle\left\langle T \phi^{\prime}\left|p(\mathbf{k}) H_{I}\right| \phi T\right\rangle}{E_{\phi T}-E_{\phi^{\prime} T}-\eta / k_{3}} .
$$

Notice that the energy being subtracted from that of the free state in the denominator is that of $p^{\dagger}(\mathbf{k})\left|\phi^{\prime} T\right\rangle$ which is an energy eigenstate of $H_{0}$. We have observed before that the state with the $q^{\dagger}|0\rangle$ is not an energy eigenstate of $H_{0}$ so what we have constructed, is it really an energy eigenstate of $H$ to order $e^{1}$ ? This we can verify readily. Acting on the above energy eigenstate with $H$, we get to order $e^{1}$

$$
\begin{aligned}
H|\phi T\rangle_{\text {pert }}= & E_{\phi T}|\phi T\rangle_{\text {pert }}+e|\phi T\rangle\left\langle T \phi\left|H_{I}\right| \phi T\right\rangle \\
& +e \sum_{\phi^{\prime}} \int \frac{\mathrm{d}^{3} \mathbf{k}}{(2 \pi)^{3}} \frac{\theta\left(k_{3}\right)}{\mathbf{k}^{2}} \frac{p^{\dagger}(\mathbf{k})\left|\phi^{\prime} T\right\rangle\left\langle T \phi^{\prime}\left|p(\mathbf{k}) H_{I}\right| \phi T\right\rangle}{E_{\phi T}-E_{\phi^{\prime} T}-\eta / k_{3}} .
\end{aligned}
$$

\footnotetext{
${ }^{3} \mathrm{By}$ suitable, we mean the bra and the ket must have the same transverse photon state since $H_{I}$ does not have those and also the scalar part which means not all scalar bra and ket combinations will be non-vanishing, but this is not essential in the present discussion. We would rather concentrate on the essential which is the $A_{L}$ part.
} 
Had we been working with a "regular" Hamiltonian and with "regular" states, the last term should not be there. Its presence is due precisely to the fact that the states with $q^{\dagger}$ above are not energy eigenstates of $H_{0}$. This means that we are not able to construct energy eigenstates of the full Hamiltonian which has all the peculiar features discussed above.

We can now return to the argument given in section 5. It seems that the physical states defined by Gauss' law cannot be written as sums of energy eigenstates due to the fact that these cannot even be constructed so that the factors $\exp ( \pm i H \delta)$ appeared in the thermal averages of any N-point function due to the time shift introduced deliberately cannot be removed. It follows that

$$
\left\langle A\left(t_{1}\right) A\left(t_{2}\right)\right\rangle \neq\left\langle A\left(t_{1}+\delta\right) A\left(t_{2}+\delta\right)\right\rangle .
$$

The inequality holds unfortunately also for the scalar field two-point function and any Npoint function. Therefore we do not have time translation invariance in the propagators and in any N-point function.

\section{Conclusion}

In this paper, we have studied the TAG formalism initiated by James and Landshoff in the abelian case. We find that in this case, the $\mathcal{O}$ operator which links the transverse states to the physical states can be solved completely and in fact, it is found to exponentiate. The exponent is the order $e^{1}$ term of the expansion of $\mathcal{O}$ in terms of the coupling. However, energy conservation is missing in those one-loop propagators which involve the longitudinal photon propagator and the $o_{1}$ vertex. This lack of energy conservation or time translation invariance can be traced back to the impossibility to construct energy eigenstates due to the rather unconventional representation of the longitudinal field and hence the Hamiltonian. Those propagators that have energy conservation at one-loop, i.e. the transverse photon propagator and the scalar propagator with an internal transverse photon line, are so only because they still do not know about the first order corrections to the free states. Their higher-loop propagators will not have energy conservation.

The source of the problem lies in the commutation relations Eq. (54) between the four annihilation and creation operators of the longitudinal field, which are, unfortunately, necessary for the field to satisfy all the canonical commutation relations and the free

field equation of motion, $\ddot{A}_{L}=0$. We do not understand why the above constraints of the Hamiltonian formulation conspire in such a way so as to forbid itself from having energy eigenstates.

What still holds in the formalism of James and Landshoff is that the longitudinal field and propagator cannot be periodic. In other words, KMS condition is not for TAG. One still knows how to construct physical states at least in the abelian theory since this 
depends only on the canonical commutation relations. However, what remains to be understood is why we cannot have a proper Hamiltonian formulation in TAG.

It must be said that the problem of energy conservation does not arise in the realtime formalism where energy is conserved everywhere. In that case, the time contour is chosen to start from $-\infty$ on the complex time plane, goes to $t=\infty$, and it ends eventually at $-\infty-i \beta$ so any time shift is easily absorbed by the infinity. Also, we only need the physical states at $t=-\infty$ which are the energy eigenstates of the free Hamiltonian, namely, the free transverse photon and scalar states since we can switch off interactions then and switch them on adiabatically.

\section{Acknowledgements}

The author would like to thank P.V. Landshoff and J.C. Taylor for discussions, N.H. Willis for some stimulating email exchanges, and the referee for pointing out a mistake

in an earlier version. The author acknowledges financial support from the Leverhulme Trust.

\section{A Appendix}

\section{A.1 $\delta$ and $\theta$ Functions}

In this appendix, we will give some relations for manipulating $\delta$ and $\theta$ functions. Given that $\theta$ has the usual meaning i.e.

$$
\begin{array}{rlr}
\theta(x)=1 & \text { for } x>0, \\
=0 & \text { for } x<0,
\end{array}
$$

then we have

$$
\int_{0}^{\beta} \mathrm{d} \tau \delta(\tau)=1
$$

Then using $\frac{\mathrm{d}}{\mathrm{d} \tau} \theta(\tau)=\delta(\tau)$, we have

$$
\theta(\beta)-\theta(0)=1
$$

so $\theta(0)=0$ has to hold for consistency.

In general, one can choose

$$
\int_{0}^{\beta} \mathrm{d} \tau \delta(\tau)=\alpha
$$

where $\alpha$ is some number, then one must use $\theta(0)=1-\alpha$ in order to be consistent. 


\section{References}

[1] S. Peigné and S.M.H. Wong, Phys. Lett. B346 (1995) 332.

[2] E. Braaten and R.D. Pisarski, Nucl. Phys. B337 (1990), 569.

[3] K. Kajantie and J. Kapusta, Ann. Phys. 160 (1985), 477.

[4] U. Heinz, K. Kajantie and T. Toimela, Ann. Phys. 176 (1987), 218.

[5] J.I. Kapusta, Finite Temperature Field Theory (Cambridge University Press, Cambridge, England, 1989).

[6] A.K. Rebhan, Phys. Rev. D48 (1993), R3967.

[7] A.K. Rebhan in Proceedings of the 3rd Workshop on Thermal Field Theories and Their Applications, Banff, Canada, August 1993, World Scientific, Edited by F.C. Khanna, R.Kobes, G. Kunstatter and H. Umezawa, p.469.

[8] A.K. Rebhan, Contributed talk at the NATO Advanced Research Workshop "Electroweak Physics and the Early Universe", 22-25 March 1994, Sintra, Portugal, hep-ph/9404292.

[9] R. Baier and O.K. Kalashnikov, Phys. Lett. B328 (1994) 450.

[10] A.K. Rebhan, Nucl. Phys. B430 (1994) 319.

[11] E. Braaten and Agustin Nieto, Northwestern University preprint NUHEP-TH-9418, August 1994.

[12] H. Nachbagauer, Z. Phys. C56 (1992) 407.

[13] K. James and P.V. Landshoff, Phys. Lett. B251 (1989), 167.

[14] P. Gaigg and M. Kreuzer, Phys. Lett. B205 (1988) 530.

[15] G. Leibbrandt, Rev. Mod. Phys. 59 (1987) 1067.

[16] P.V. Landshoff, Phys. Lett. B227 (1989), 427.

[17] K.A. James in Proceedings of the Workshop of Physical and Nonstandard Gauges, Vienna, Austria, September 19-23, 1989, Edited by P. Gaigg, W.E. Kummer, M. Schweda, Springer-Verlag, 1990, p.303.

[18] K.A. James, Z. Phys. C48 (1990) 169.

[19] K.A. James, Z. Phys. C49 (1991) 115.

[20] N.P. Landsman and Ch.G.van Weert, Phys. Rep. 145 (1987) 141. 


\section{B Figure Captions}

Fig. 1 Graphs contributing to part of the one-loop scalar propagator with $\theta\left(q_{3}\right)$. The loop consists of one longitudinal and one scalar line.

Fig. 2 Graphs contributing to part of the one-loop longitudinal propagator with $\theta\left(k_{3}\right)$. The loop consists of scalar lines.

Fig. 3 The tadpole contribution to the one-loop longitudinal propagator. This graph is energy conserved. 
This figure "fig1-1.png" is available in "png" format from: http://arxiv.org/ps/hep-th/9501032v3 
This figure "fig1-2.png" is available in "png" format from: http://arxiv.org/ps/hep-th/9501032v3 